\title{
Bayesian Meta-Analysis with Weakly Informative Prior Distributions
}

\author{
Donald R. Williams \\ University of California, Davis \\ Philippe Rast \\ University of California, Davis \\ Paul-Christian Bürkner \\ Institute of Psychology, University of Münster
}

\begin{abstract}
Developing meta-analytic methods is an important goal for psychological science. When there are few studies in particular, commonly used methods have several limitations, most notably of which is underestimating between-study variability. Although Bayesian methods are often recommended for small sample situations, their performance has not been thoroughly examined in the context of meta-analysis. Here, we characterize and apply weaklyinformative priors for estimating meta-analytic models and demonstrate with extensive simulations that fully Bayesian methods overcome boundary estimates of exactly zero betweenstudy variance, better maintain error rates, and have lower frequentist risk according to Kullback-Leibler divergence. While our results also show that combining evidence with few studies is non-trivial, we argue that this is an important goal that deserves further consideration in psychology. Further, we suggest that frequentist properties can provide important information for Bayesian modeling. We conclude with meta-analytic guidelines for applied researchers that can be implemented with the provided computer code.
\end{abstract}

Keywords: Bayesian, Meta-analysis, Weakly informative priors, small sample

\section{Introduction}

An important goal for psychological science is developing quantitative methods that can combine evidence from multiple studies (Carlsson, Schimmack, Williams, \& Bürkner, 2017; Scheibehenne, Jamil, \& Wagenmakers, 2016; Schmidt, 1992). When there are few studies in particular, this allows for synthesizing evidence in the beginning stages of a research question which can provide important information for future inquiry (Van Aert \& Van Assen, 2017). However, even for registered replications, there is often heterogeneity due to experimental design, manipulation properties, and environmental context (e.g., varying cultures or country of origin) (Klein et al., 2014; O’Donnell et al., 2017). Because of these potential between-study differences, the analytical decision is often posited as a choice between a

Research reported in this publication was supported by three funding sources: (1) The National Academies of Sciences, Engineering, and Medicine FORD foundation pre-doctoral fellowship to DRW; (2) The National Science Foundation Graduate Research Fellowship to DRW; and (3) the National Institute On Aging of the National Institutes of Health under Award Number R01AG050720 to PR. The content is solely the responsibility of the authors and does not necessarily represent the official views of the National Academies of Sciences, Engineering, and Medicine, the National Science Foundation, or the National Institutes of Health. fixed-effects (FE) or random-effects (RE) model (Schmidt, Oh, \& Hayes, 2009). The former assumes the effect-size is a fixed unknown quantity and allows for conditional inference about the set of $k$ studies (Viechtbauer, 2007), even in the presence of heterogeneity (Hedges \& Vevea, 1998; Schmidt, 2008). In contrast, the RE model answers a distinct question that allows for unconditional ${ }^{1}$ inferences about the average effect in a population of studies, of which the $k$ studies are assumed to be a random sample (Curran \& Hussong, 2009; Hedges \& Vevea, 1998).

In other words, in contrast to common thought (Rice, Higgins, \& Lumley, 2017), both the FE and RE models can provide valid inference in the presence of heterogeneity, but differ in the questions that they answer (Overton, 1998). However, when using the RE model to make the arguably richer unconditional inference, there are several statistical issues that can arise. For example, there is a large body of work in medical statistics documenting difficulties in estimating the between-study variance $\tau^{2}$ (Veroniki et al., 2016). This has resulted in the development of a number of alternative estimators, such as DerSimonian and Laird (Dersimonian \& Laird, 1986), Hunter and Smith (Hunter \& Schmidt, 2004), and Sidik and Jonkman (Sidik \& Jonkman, 2005). However, even the most common and well described estimators of $\tau^{2}$ can provide biased estimates under suboptimal conditions

\footnotetext{
${ }^{1}$ By unconditional we refer to inferences that can generalize beyond the observed studies.
} 
(e.g., few studies). For example, Chung, Rabe-Hesketh, and Choi (2013) showed in a simulation study that a large proportion of the returned values for $\tau^{2}$ were estimated as exactly zero (i.e., boundary estimates) when the true value was positive. This was a common finding for cases where the metaanalysis comprised a limited number of studies (i.e., $k=5$ to 10 studies) even when the true value for $\tau^{2}$ was large. This suggests that, when we observe a zero estimate in practice, it does not necessarily reflect the absence of between-study variance but it is possibly a result of an estimation issue.

Importantly, this can influence inference regarding the summary effect size estimate $\mu$ (Rhodes et al., 2016). Since $\tau^{2}$ is a source of variation, underestimation or a boundary estimate results in an inaccurate summary estimate. Namely, too small variance estimates typically result in liberal estimates of $\mu$, as the confidence intervals are too narrow (Hunter \& Schmidt, 2000). That is, even for a random effects model (Guolo \& Varin, 2017), type I error rates and coverage probabilities for meta-analytic estimates are not necessarily at expected levels (Chung, Rabe-Hesketh, \& Choi, 2013; Hedges \& Vevea, 1998; Rhodes et al., 2016) ${ }^{2}$. With few studies in particular, $95 \%$ confidence intervals can have coverage probabilities in the $80 \%$ 's (Brockwell \& Gordon, 2001).

To overcome these challenges, Bayesian methods are often applied for meta-analysis, particularly for estimating between-study heterogeneity when the number of primary studies is small (Friede, Röver, Wandel, \& Neuenschwander, 2017; Rhodes et al., 2016). In fact, as summarized in McNeish (2016), Bayesian methods are often recommended with small samples sizes in the behavioral sciences. Compared to classical ${ }^{3}$ approaches, Bayesian methods offer several potential advantages: Estimation with Markov chain Monte Carlo (MCMC) ensures that $\tau^{2}$ is not estimated at the boundary of the sample space (Gelman et al., 2014). This occurs because MCMC sampling for variance components is restricted to positive values and explores the whole (posterior) distribution of the parameter and not just the mode. This guarantees non-zero variances. In contrast, classical estimators can estimate negative values, which are then set to zero because of a lack of alternative options (Kolenikov \& Bollen, 2012; Viechtbauer, 2007)

However, Bayesian meta-analytic approaches remain relatively uncommon in practice and, in simulation work, it was shown that they tend to have the opposite problem of the classical estimators: $\tau^{2}$ is often overestimated resulting in a conservative estimate for the summary effect (Lambert, Sutton, Burton, Abrams, \& Jones, 2005; Rhodes, Turner, \& Higgins, 2015). The approaches adopted in the Bayesian literature have ranged from approximate (modal estimation) methods to using informative priors (Chung, Rabe-Hesketh, \& Choi, 2013; Lambert et al., 2005; Rhodes et al., 2016). Importantly, when Bayesian methods have been shown to outperform classical methods, informative priors are often de- rived from subject-specific expertise or directly from previous studies (McNeish, 2016). Because specific prior information is not always available, an important goal is to explore prior distributions that are not only informative but also general enough to apply to many situations. This class of prior is referred to as weakly informative (Gelman, 2006; Gelman, Simpson, \& Betancourt, 2017) and, in the context of psychological meta-analyses, has not been examined with fully Bayesian methods.

In the present paper, we aim to bring awareness to methodological issues for estimating $\tau^{2}$ in meta-analysis. This topic has received little consideration in the psychological literature, but has serious implications for cumulative science (Kenny \& Judd, 2017). The estimation issues are most pronounced with few studies, for example, and this can hinder ongoing replication efforts that rely on meta-analysis to synthesize a potentially small number of studies (Garrison, Finley, \& Schmeichel, 2017; Gronau et al., 2017). To address this issue, we first describe the random-effects metaanalytic model and classical methods used to estimate $\tau^{2}$. Next, Bayesian methods for meta-analysis are introduced where we focus on the various proposed prior distributions for $\tau^{2}$ and describe the use of weakly informative priors. To make the topic more tangible, we use a simple simulation and real data to demonstrate that these issues are not just a statistical curiosity. Then, with extensive numerical experiments we compare frequentist properties between classical and Bayesian estimators of between-study variance and the meta-analytic summary estimates. We end by providing guidelines for fitting random effect meta-analyses in practice, where we emphasize advantages of Bayesian methods.

\section{Random-Effects Model}

Meta-analytic methods are the most common way to synthesize evidence (Borenstein, Hedges, Higgins, \& Rothstein, 2009), and are considered to provide the most accurate effect estimate given the covered literature (Haidich, 2010). However, empirical studies on the same topic are often heterogeneous with respect to sample characteristics and measures (Higgins \& Thompson, 2002). Thus, the quality of inference depends on incorporating between-study variance into estimating the summary effect. The random-effects model allows for estimating an average effect from heterogeneous studies (Raudenbush, 2009), while also accounting for between-study variance $\left(\tau^{2}\right)$ and allowing for generalizable inferences (Hedges \& Vevea, 1998). This can provide nominal frequentist properties-coverage probabilities and error

\footnotetext{
${ }^{2}$ It should be noted that we do not think type I error is the best concept to base statistical inference. However, we take the pragmatic position that most researchers in psychology currently do not yet share this belief.

${ }^{3}$ We avoid the term frequentist, because all methods and statistics can be evaluated with respect to frequency properties.
} 
rates-for the meta-analytic effect $\mu$. Some have suggested that zero between-study variance is untenable (Brockwell \& Gordon, 2001), and it is more reasonable to always assume a random effect model that is defined as

$$
\begin{aligned}
y_{i} & \sim \mathcal{N}\left(\delta_{i}, \sigma_{i}^{2}\right), \\
\delta_{i} & \sim \mathcal{N}\left(\mu, \tau^{2}\right), \\
i & \in\{1, \ldots, k\},
\end{aligned}
$$

where $i$ indexes the individual study. Here, $y_{i}$ is the observed effect size, $\delta_{i}$ is the latent true effect size, respectively, and $\sigma_{i}^{2}$ is the variance from the $i$ th study. The study-specific effects are assumed to share a common distribution with mean $\mu$ and variance $\tau^{2}$. Accordingly, the random-effects model has two sources of variation $\sigma_{i}^{2}$ and $\tau^{2}$, each of which contributes to the confidence interval for $\mu$. A common approach uses a $(1-\alpha) 100 \%$ Wald-type interval as

$$
\hat{\mu} \pm z_{1-\alpha / 2}\left(\sum_{i}\left(\sigma_{i}^{2}+\tau^{2}\right)^{-1}\right)^{-\frac{1}{2}}
$$

where $\sigma_{i}^{2}$ is generally obtained from the individual studies and are assumed to be known (i.e. measured without error), while $\tau^{2}$ is a model parameter that has to be estimated (Chung, Rabe-Hesketh, \& Choi, 2013). From Equation (2), it is clear that inferences about $\mu$ depend critically upon estimating $\tau^{2}$ accurately.

\section{Classical Estimators}

The DerSimonian and Laird (DL) method is the default estimator for $\tau^{2}$ in commonly used software such as, for example, Comprehensive Meta-Analysis (Borenstein, Hedges, Higgins, \& Rothstein, 2005; Dersimonian \& Laird, 1986). However, Veroniki et al. (2016) recently reviewed 16 alternative methods for estimating $\tau^{2}$ and provided a qualitative recommendation to use restricted maximum likelihood (REML) instead of DL, but noted further simulations were needed. In the present paper, due to popularity and current recommendations, we consider the DL and REML estimators for $\tau^{2}$.

\section{DerSimonian and Laird}

The DL method is a non-iterative approach that is based on Cochran's Q-statistic (Dersimonian \& Laird, 1986). The $Q$ statistics provides a measure of discrepancy between subject-specific effects and the pooled estimate from a fixed effect $\left(\hat{\mu}_{F E}\right)$ analysis

$$
Q=\sum_{i=1}^{k} \frac{\left(y_{i}-\hat{\mu}_{F E}\right)^{2}}{\sigma_{i}^{2}}
$$

Here, the sum of the $i$ th squared difference between the study-specific effect $y_{k}$ and $\hat{\mu}_{F E}$ are divided by the study variance $\sigma_{k}^{2}$. The DL estimator of $\tau^{2}$ is then obtained with

$$
\tau_{D L}^{2}=\max \left\{0, \frac{Q-(k-1)}{\sum w_{i, F E}-\frac{\sum w_{i, F E}^{2}}{\sum w_{i, F E}}}\right\},
$$

where $\sum w_{i, F E}$ denotes the sum of the study weights $w_{i, F E}=$ $\frac{1}{\sigma_{i}^{2}}$ from a fixed-effect meta-analysis. In simulations, the DL estimator was shown to have adequate frequentist properties when $k$ is large and the values for $\tau^{2}$ are very small (Sidik \& Jonkman, 2007). With increasing values of $\tau^{2}$, however, the DL estimator can show substantial downward bias which then affects the coverage properties of $\mu$ (Equation: 5) (Chung, Rabe-Hesketh, \& Choi, 2013).

\section{Restricted Maximum Likelihood}

In contrast to the DL method, REML is an iterative approach that is not specific to meta-analysis and is commonly used in multilevel models (Bates, Mächler, Bolker, \& Walker, 2014), of which a random-effects meta-analysis is a special case. Indeed, REML is often preferred over maximum likelihood (ML), because random effect variances show less downward bias (Raudenbush \& Bryk, 2002). The REML estimator $\left(\tau_{R E M L}^{2}\right)$ is computed with a two-step approach, in which the first obtains maximum likelihood estimates $\tau_{M L}^{2}$ and $\mu_{M L}^{2}$. These then serve as initial estimates for the following

$$
\tau_{R E M L}^{2}=\max \left\{0, \frac{\sum w_{i, R E}\left(\left[\delta_{i}-\hat{\mu}_{R E M L}\right]\right)-\sigma_{i}^{2}}{\sum w_{i, R E}}+\frac{1}{\sum w_{i, R E}}\right\},
$$

with the random effect study weights as $\frac{1}{\delta_{i}+\tau_{R E M L}^{2}}$. Importantly, this two-step procedure ensures the initial estimate is greater than zero, and that each step produces a nonnegative value (Veroniki et al., 2016). A number of studies have shown that the REML method shows less downward bias in the estimate of between study variance than the DL estimator (Chung, Rabe-Hesketh, \& Choi, 2013; Sidik \& Jonkman, 2007), although both estimators produce similar proportions of boundary estimates (Chung, Rabe-Hesketh, \& Choi, 2013).

\section{Bayesian Meta-Analysis}

Bayesian methods provide an alternative to classical approaches (Higgins \& Spiegelhalter, 2002; Sutton, Abrams, \& Health, 2001). They are often recommended for estimating parameters on the boundary of the sample space and when samples are small (Baldwin et al., 2005; McNeish, 2016). For example, Bayesian methods ensure non-boundary estimates ( $\tau^{2}>0$; Chung, Rabe-Hesketh, Dorie, Gelman, \& Liu, 2013) and can thus guard against liberal estimates for the summary effect (Chung, Rabe-Hesketh, \& Choi, 2013), as well as estimating the full posterior distribution of $\tau^{2}$. A Bayesian model begins with specifying a joint probability distribution 
(Gelman et al., 2014). For a summary statistic $y_{i}(i \in\{1, \ldots, k\})$ generated from a normal distribution with a study-specific mean $\delta_{i}$ and variance $\sigma_{i}^{2}$, Bayes theorem states that

$$
p(\boldsymbol{\theta} \mid y) \propto p(y \mid \boldsymbol{\theta}) p(\boldsymbol{\theta})=\prod_{i=1}^{k} p\left(y_{i} \mid \delta_{i}, \sigma_{i}^{2}\right) p\left(\delta_{i} \mid \mu, \tau^{2}\right) p\left(\mu, \tau^{2}\right)
$$

with the parameters $\boldsymbol{\theta}=\left(\delta_{1}, \ldots, \delta_{k}, \mu, \tau^{2}\right): \mu$ is the population average, $\delta_{i}$ is the study-specific effect, and $\tau^{2}$ is the between study variance. In meta-analytic notation, the Bayesian model follows

$$
\begin{aligned}
y_{i} & \sim \mathcal{N}\left(\delta_{i}, \sigma_{i}^{2}\right) \\
\delta_{i} & \sim \mathcal{N}\left(\mu, \tau^{2}\right) \\
\left(\mu, \tau^{2}\right) & \sim p(.), \tau^{2}>0 .
\end{aligned}
$$

While this closely resembles a classical model, there are notable differences, such as the definition of prior distributions $p\left(\right.$.) for $\mu$ and $\tau^{2}$, in addition to restricting $\tau^{2}$ to positive values.

\section{Prior Distributions}

The prior distribution is a controversial concept in Bayesian statistics that has been source of many debates (Berger, 2003; Gelman, Jakulin, Pittau, \& Su, 2008). Objections are often raised that incorporating prior information into a statistical model is subjective (Goldstein, 2006). As such, certain priors are often assumed so that Bayesian and likelihood only methods produce similar estimates (Berger, 2006). Indeed, when assuming an improper uniform prior distribution (a prior which puts equal weight on all possible parameter values), the likelihood is proportional to the posterior distribution. However, for estimating variances in particular, uniform prior distributions are not generally recommended as they give too much prior weight to unreasonably large values (Gelman, 2006). In other words, the notion of an non-informative prior is a misnomer, as it can be very informative (Gelman et al., 2017). For example, data in psychological meta-analyses are generally on a standardized scale (e.g., Cohen's d, Fisher's z, etc.). Assuming the $d$ scale for $y_{i}$, this suggests that $\tau^{2}>0.25$ and $\mu>1.0$ are unlikely to occur. This information is useful and can be formally incorporated into a Bayesian meta-analytic model. To be clear, we are not suggesting to include unverifiable subjectivity into an analysis. Rather, just like the assumed likelihood (e.g., Gaussian) or other assumptions (e.g., homogeneity of variances), the prior can be viewed as a verifiable component of the model and is open to criticism (Gelman, 2013). We can thus examine how a proposed prior distribution influences performance measures such as bias and the variance of our estimators.

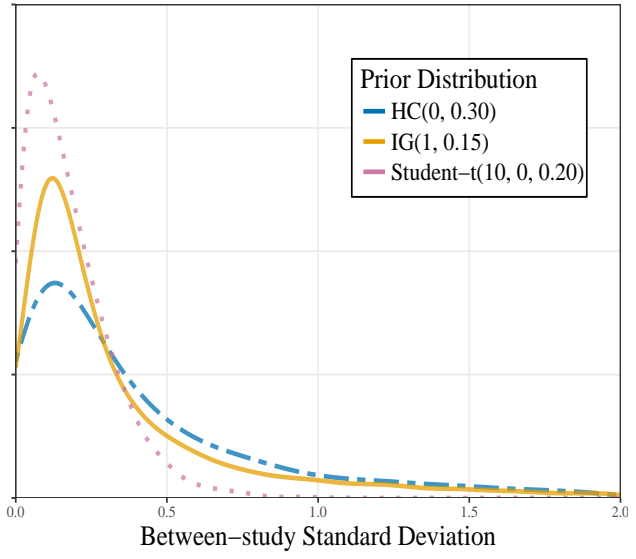

Figure 1. Probability densities for three types of prior distributions, the half-Cauchy (HC), the inverse-gamma (IG), and the Student- $t^{+}$. The heavy tails of HC allows for the possibility of large values, which provides a general prior distribution for many applications.

In clinically oriented fields, substantial effort has been invested into deriving an informed prior distribution for between-study variances from empirical studies (Pullenayegum, 2011; Rhodes et al., 2015; Turner, Davey, Clarke, Thompson, \& Higgins, 2012). Recently, van Erp, Verhagen, Grasman, and Wagenmakers (2013) extracted estimates of between-study heterogeneity from psychological literature. This consisted in obtaining estimates of $\tau$ from metaanalyses ranging from $1990-2013$. Based on this distribution of estimates, van Erp et al. (2013) recommended the following prior distribution as a default for psychology

$$
\tau \sim \text { inverse-gamma }(\alpha=1, \beta=0.15),
$$

where $\alpha$ and $\beta$ denote the shape and scale parameters of the inverse-gamma $(I \mathcal{I})$ distribution. However, the recommendation of van Erp et al. (2013) and Gronau et al. (2017) did not include verification (e.g. with simulations) of performance. This is unfortunate, as this prior may be problematic for meta-analysis in particular (Polson \& Scott, 2012): It was shown to have undesirable characteristics when on the boundary of the sample space, as the parameter estimates can be especially sensitive to the values for $\alpha$ and $\beta$ (Asparouhov \& Muthén, 2010; Gelman, 2006). Due to its conjugate properties, the inverse-gamma prior is generally used for computational convenience that enables efficient Gibbs samplings (Gelman, 2006; Gelman \& Dyk, 2007), which is probably the reason, why this prior family was chosen by van Erp et al. (2013) in the first place (with parameter values adjusted according to the obtained effect sizes). The Gibbs sampler has largely been superseded by more efficient methods such as the Hamiltonian Monte Carlo (HMC) NO-UTurn Sampler (used in this present paper) (Betancourt, 2017; Hoffman \& Gelman, 2014). This allows not only for more 
flexibility in prior distributions, but also more stable parameter estimates and faster computation-especially in the case of multilevel problems with high correlations among estimated parameters (Monnahan, Thorson, \& Branch, 2017; Zhang \& Sutton, 2014).

Moreover, a prior does not necessarily have to reflect historical or empirical data, but can be chosen based on desirable mathematical properties. Here the goal is a weakly informative prior (Gelman, 2006; Gelman et al., 2017): a middle ground between a fully informative and "uninformative" (flat) prior distribution. For variance components, the folded- $t$ family provides such a trade-off.

$$
\tau \sim \text { Student }-t^{+}\left(x_{o}, s, v\right),
$$

where $x_{o}$ is the location, $s$ is the scale, and $v$ is the degrees of freedom parameter that controls the tail-heaviness. In the vast majority of cases, we should set $x_{0}=0$ so that the folded- $t$ distribution becomes the half- $t$ distribution, which can take on all positive values only. As $v \rightarrow \infty$ the distribution approaches normality (i.e. a folded normal distribution). This mathematical property provides the ability to control the tail-regions and this allows for specifying a prior that is both informative and generic. As a special case, for example, the half-Cauchy $(\mathcal{H C})$ distribution $\left(x_{o}=0, v=1\right)$ coincides with the Student- $t^{+}$distribution with one degree of freedom. This distribution has very heavy tail regions which allows for the possibility of large values (Figure 1). This prior in particular has been recommend for variance components (Gelman, 2006) and was also shown to perform well in terms of frequentist risk (Polson \& Scott, 2012). This provides several benefits for applied researchers conducting meta-analyses. For example, this prior can reflect that the distribution of $\tau^{2}$ can only be positive, is often right skewed (van Erp et al., 2013), and that smaller values are more plausible than large values (Rhodes et al., 2015). However, the heavy-tailedness ensures that these assumptions are not too strong and can apply to many situations (i.e., a middle-ground).

While there are examples in applied settings (Bürkner, Williams, Simmons, \& Woolley, 2017; Williams \& Bürkner, 2017), the performance of weakly informative priorsspecifically folded- $t$ distributions-has not been characterized in the context of meta-analysis. However, finding an optimal estimator for $\tau^{2}$ is important for several reasons. In psychology, meta-analyses are often performed with few studies and inference (if relying on interval exclusion of 0 ) can largely depend on the estimator used (Gronau et al., 2017; Scheibehenne et al., 2016). Second, developing methods for use in the beginning stages of a research program (when there are few studies) can improve cumulative science. We thus examine the recommended inverse-gamma compared to weakly informative priors, in addition to the previously described classical estimators (DL and REML). For clarity, we first demonstrate how boundary issues can influence estimation and inference with two motivating examples.

\section{Boundary Issues}

\section{Motivating Example: Simulation}

This section provides an example to illustrate the issue of estimating parameters at their boundary. We also now refer to between-study standard deviation $\tau$ instead of the variance $\tau^{2}$, as this corresponds to the parameterizations of the fitted Bayesian models. A meta-analysis scenario was simulated (with 500 simulation trials) with generic values for $\mu=0$ and $\tau=0.15$. We assumed the sampling distribution for $\sigma_{i}^{2}$ is known and follows a uniform distribution $\sigma_{i}^{2} \sim \mathcal{U}(0.05,0.35)$. We then computed the sampling distribution for $\hat{\tau}$ with the classical estimators (DL and REML) and Bayesian methods using two prior distributions: (1) the recommended inverse-gamma distribution (Equation 8) and (2) a half-Cauchy distribution where $x_{o}=0, s=0.3$, and $v=1$ (Equation 9). This value for $s$ was based on both prior information about reasonable values for $\tau$ and knowledge of the Cauchy distribution (Figure 2). Specifically, this prior assumes there is a $50 \%$ prior probability that values of $\tau$ will be less than 0.3 . To demonstrate how estimating betweenstudy heterogeneity influences $\mu$, we also computed the distribution of $p$-values which should be uniform for this example $(\mu=0)$. For comparative purposes, we approximated a two-sided Bayesian $p$-value from the posterior distribution for $\mu$ as

$$
p \text {-value }=\min \{p(\mu>0 \mid y, \mathcal{M}), p(\mu<0 \mid y, \mathcal{M})\} \times 2
$$

where the minimum directional posterior probability, conditional on the data $y$ and fitted model $\mathcal{M}$, is multiplied by 2 . This approximation is justified by the relationship between posterior probabilities and one-tailed $p$-values (Greenland \& Poole, 2013), although it requires very specific assumptions (Gelman, 2013).

The results are presented in Figure 2, where panels A show the sampling distributions and panels $\mathrm{B}$ the distribution of $p$-values. The boundary issue is clearly seen with the DL and REML estimator. For example, the sampling distribution of $\tau$ is bimodal with considerable density at zero. Indeed, for both estimators, a large proportion of the returned values were exactly zero $(\mathrm{DL}=31 \%$ and $\mathrm{REML}=25 \%)$. In contrast, the Bayesian models produced a unimodal sampling distribution in which the returned values show consistent right-skew. The boundary issue is not just of statistical interest. Figure 2 shows the distribution of $p$-values for the summary effect $\mu$, which should be uniform under the null hypothesis of no effect. The classical estimators showed an increase in small $p$-values such that error rates exceeded 

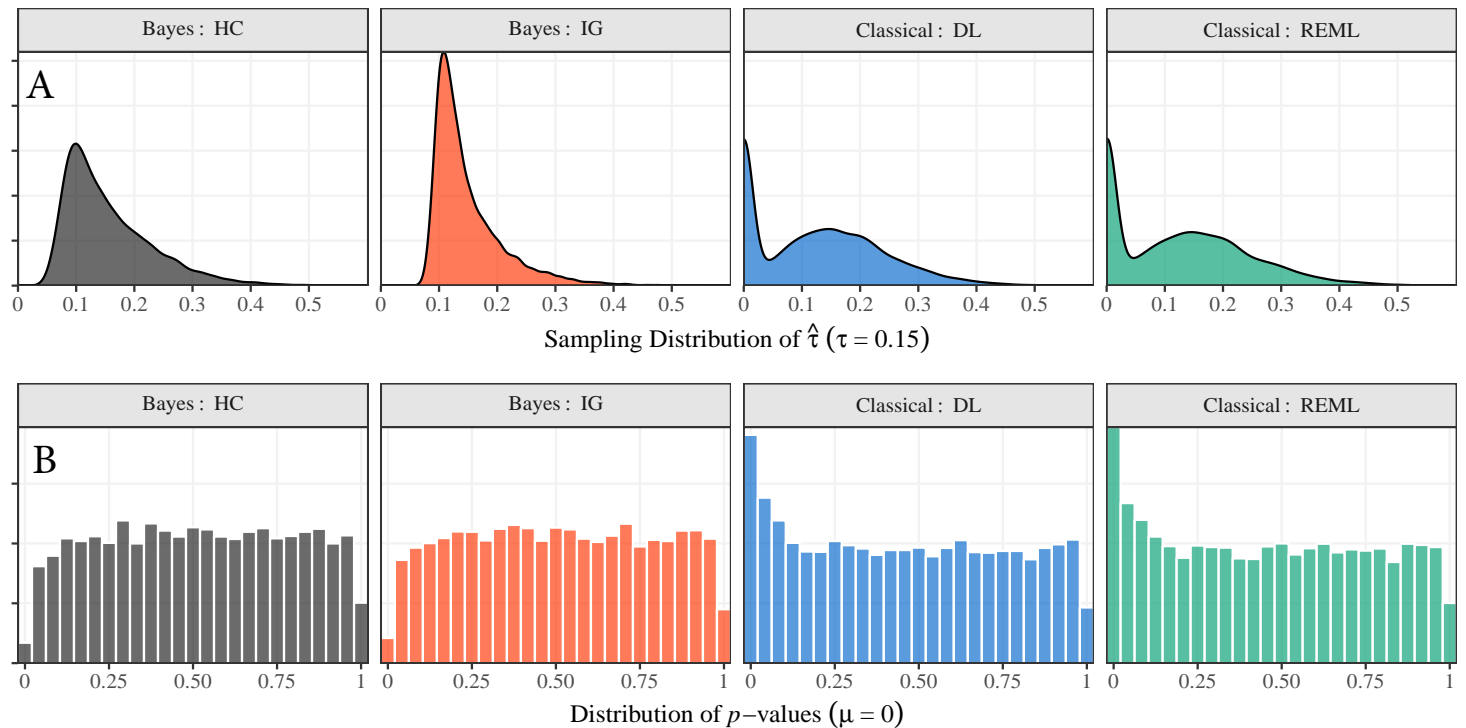

Figure 2. A: Sampling distribution of $\tau$. The classical methods (DL = DerSimonian and Laird; REML = Restricted Maximum Likelihood) are caught on boundary of sample space, as indicated with the bimodality. The Bayesian models (HC = Half-Cauchy; IG = Inverse-gamma) provided all non-zero estimates. B: Distribution of p-values under the null hypothesis $(\mu=0)$, which should be uniform. Underestimation of $\tau$ is resulting in to many small p-values $(<0.05)$ for the classical methods.

nominal levels. This occurs because, when $\tau$ is estimated as zero, we effectively fit a fixed effects model. Notably, the Bayesian $p$-value shows the opposite pattern. There are not enough $p$-values below 0.05 . Since $1-\alpha$ is coverage for 0 , this suggests the true value was covered more often than expected.

\section{Motivating Example: Real Data}

We analyzed two data sets that can be found in the $\mathrm{R}$ package metaBMA (Heck, Gronau, \& Wagenmakers, 2017). The first reports the effects of social norms on towel reuse in hotels $(k=7)$, whereas the second is from a recent metaanalysis on power pose experiments $(k=6)$. We estimated random-effects models described in Equations (1) and (7) . The individual effects $y_{i}$ for both data sets were analyzed on Cohen's $d$ scale. As seen in Table 1, boundary estimates of zero are present for both the DL and REML estimators. For the towel reuse analysis, DL estimated $\tau$ as 0.07 and the REML estimate was on the boundary of the sample space $(\tau=0)$. For the power-pose data, both estimators returned values of zero which would lead to the inference of zero variability between studies. In contrast, the Bayesian models always estimated positive values for $\tau$. There were differences between the posteriors, however, with the halfCauchy prior consistently producing both smaller point estimates and wider probability intervals than the inversegamma prior. The Bayesian intervals for the summary effect $\mu$ were always wider than those obtained with the DL and
REML estimators. For the towel reuse analysis, the Bayesian intervals for $\mu$ included zero, whereas the frequentist intervals excluded zero. That is, there was inconsistent evidence for what is traditionally used to argue for the presence of an effect.

\section{Simulation Study}

The above motivating examples clearly demonstrated issues that can arise in meta-analysis, and how the estimates can differ more than one might expect. We now perform extensive numerical experiments to fully characterize performance between methods. Rather than assuming known sampling distributions for $\sigma_{i}^{2}(i \in\{1, \ldots, k\})$, we instead generated individual studies with the following steps:

1. Generate $k$ effect sizes $\delta_{i}$ from $\mathcal{N}(\mu, \tau)$, where $k \in(3$, $5,10,20$, and 40$), \mu \in(0$ and 0.4$)$ and $\tau \in(0,0.10,0.20$, $0.30,0.50$, and 0.60$)$.

2. Randomly select $k$ sample sizes $\{N \mid l \leq N \leq u\}$ with lower $l$ and upper bounds $u$ as 10-22, 22-78, and 78122.

3. Generate $k$ studies consisting of two groups of $N$ size as $y_{1} \sim \mathcal{N}\left(\delta_{i}, 1\right)$ and $y_{2} \sim \mathcal{N}(0,1)$.

4. For the $i$ th study $\in(1, \ldots, k)$, compute the summary statistics $y_{i}$ and $\sigma_{i}^{2}$.

5. For each set of $k$ studies, estimate the meta-analytic model following Equations (1) and (7).

The sample sizes and effect size $(d=0.4)$ corresponded to sets of studies with average power of $20 \%, 50 \%$, and $80 \%$. 
Table 1

Meta-analytic models fitted to psychological data, in which 3 of 4 of the classical estimates for $\tau$ are zero. For the Towel Reuse data in particular, the Bayesian (HC = Half-Cauchy; IG = Inverse-gamma) and classical models (DL = DerSimonian and Laird; $R E M L=$ Restricted Maximum Likelihood) would lead to opposing inferences for the summary effect, assuming the decision rule is interval exclusion of zero. CI denotes confidence intervals for the classical methods and highest density intervals for the Bayesian methods.

\begin{tabular}{|c|c|c|c|c|c|c|c|c|}
\hline \multirow[b]{2}{*}{ Model } & \multicolumn{4}{|c|}{ Towel Reuse } & \multicolumn{4}{|c|}{ Power Pose } \\
\hline & Cohen's $d$ & $95 \% \mathrm{CI}$ & $\tau$ & $95 \% \mathrm{CI}$ & Cohen's $d$ & $95 \% \mathrm{CI}$ & $\tau$ & $95 \% \mathrm{CI}$ \\
\hline \multicolumn{9}{|l|}{ Bayesian } \\
\hline$\overline{\mathcal{H C}(0,0.3)}$ & 0.11 & {$[-0.05,0.22]$} & 0.07 & {$[0.00$,} & 0.22 & {$[0.07,0.36]$} & 0.06 & {$[0.00$} \\
\hline $\mathcal{I G}(1,0.15)$ & 0.10 & {$[-0.04,0.23]$} & 0.09 & {$[0.02,0.23]$} & 0.22 & {$[0.07,0.37]$} & 0.08 & {$[0.02,0.20]$} \\
\hline \multicolumn{9}{|l|}{ Classical } \\
\hline $\mathrm{DL}$ & 0.11 & {$[0.01,0.22]$} & 0.07 & {$[0.00,0.69]$} & 0.22 & {$[0.10,0.34]$} & 0.00 & {$[0.00,0.10]$} \\
\hline REML & 0.12 & {$[0.04,0.21]$} & 0.00 & {$[0.00,0.69]$} & 0.22 & {$[0.10,0.34]$} & 0.00 & {$[0.00,0.10]$} \\
\hline
\end{tabular}

This value was chosen because it corresponds closely to the median observed effect size in psychology.

For the classical estimators, we used Wald-type intervals for the summary effect $\mu$, from which coverage and type I error rates were computed. We fitted four Bayesian models that differed in the prior distributions for $\tau$. The first two used $\mathcal{H} C$ with $x_{o}=0$ and two values for the scale $s(0.3$, and 0.5$)$. We also used another half- $t$ prior but with lighter tails $\left(v=10, x_{o}=0, s=0.2\right)$. For this prior in particular, we expected poor performance at large values of $\tau$. The final Bayesian model was fitted with the inverse-gamma described in Equation (8). To improve sampling efficiency, we used a diffuse prior on $\mu \sim \mathcal{N}(0,1)$ which presumes a $95 \%$ prior probability the value is within -2 and 2 on $d$ scale. Each model consisted of two-chains of 1,000 post-warm-up posterior samples each (after excluding 1,000 iteration of warmup per chain). The posterior distributions were summarized with medians and $95 \%$ highest density intervals. Before beginning the simulation, we determined convergence by examining model diagnostics (e.g., $\hat{R}$, effective sample size, visual inspection) for several different conditions.

All computations were done in $\mathrm{R}$ and included 1,000 simulation trials for each condition. We used the package metafor for the classical models (Viechtbauer, 2010), whereas the Bayesian meta-analytic models were fitted with the probabilistic programming language Stan (Stan Development Team, 2015) that employs a modern Markov chain Monte Carlo algorithm (Hamiltonian Monte Carlo with the No-U-Turn Sampler). The highest density intervals were obtained with HDInterval (Meredith \& Kruschke, 2016) (https://osf.io/9n4zp/)

\section{Results}

\section{Between-study Variation}

Boundary Estimates. For each simulation condition, we computed the proportion of estimated values $\hat{\tau}$ that were exactly zero as

$$
P=\frac{1}{n} \sum_{i=1}^{n}\left\{\begin{array}{ll}
0 & \text { if } \hat{\tau}>0 \\
1 & \text { if } \hat{\tau}=0
\end{array}\right\}
$$

where $n$ is the number of simulation trials. The results are presented in Figure 3 (panel A). Here we see the Bayesian models estimated all values as positive and thus obtained no boundary estimates. Of course, in case of $\tau=0$, this is not an ideal result, because the fitted models suggest existent between-study variation. However, it has to be noted that, for $\tau=0$, REML and DL both estimated many positive values for all conditions, as well. The largest proportion of positive estimates was obtained with $k$ of 40 . For positive values of $\tau(>0)$, the classical models showed clear boundary issues which coincides with the motivating example based on simulation (Figure 2). While this issue was most pronounced with the smaller values for $k$, boundary estimates of zero were observed for all conditions, but with declining proportions for large values of $\tau$. Moreover, when the primary studies consisted of small sample sizes (Small N: average power of $20 \%$ to detect $d=0.4$ ), boundary estimates persisted with the largest value for $k$. In fact, with 40 studies included in the meta-analyses and the median observed value for $\tau(0.20)$ reported in van Erp et al. (2013), DL estimated $20 \%$ of the values as zero, whereas REML provided a slightly smaller proportion of boundary estimates.

Loss Functions. We approached evaluating the competing models as a decision problem. That is, given a set of estimators, one must decide which to use in practice but also understand the associated risk inherent to all estimation problems. We thus draw upon decision theory and use loss functions for assessing the frequentist (i.e., averaged across the sampling distribution) risk associated with each estimate. With the parameter space $\Theta$ consisting of true values for $\theta$, we considered two loss functions, the first of which 


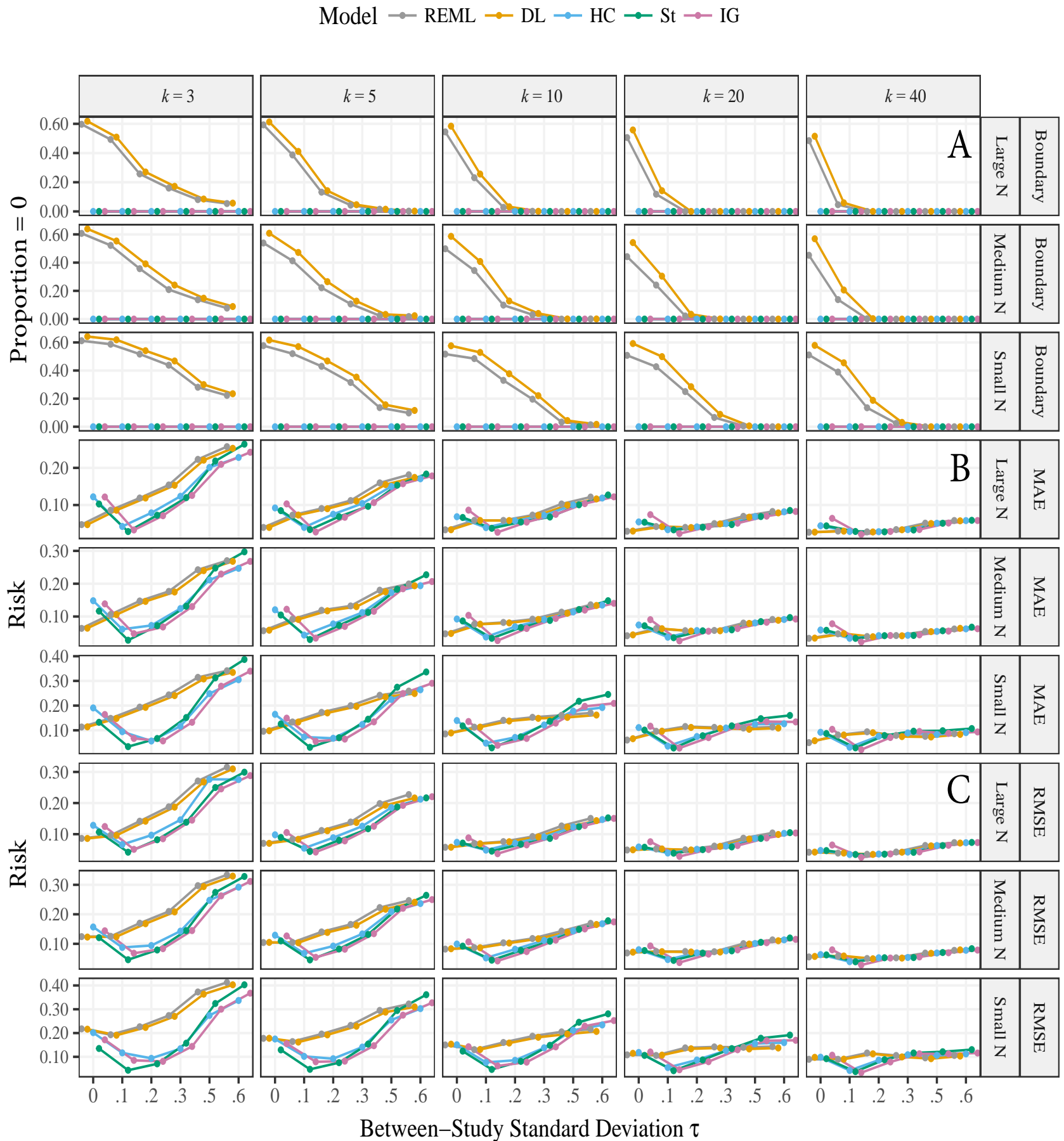

Figure 3. A: Boundary estimates (proportion $\tau=0$ ). B: Mean absolute error. C: Root mean squared error. DL $=$ DerSimonian and Laird; REML = Restricted Maximum Likelihood; HC = Half-Cauchy; St $=$ Student-t; IG = Inverse-gamma. The total studies included in each meta-analysis is denoted with $k$. The sample sizes in the primary studies correspond to average power of $20 \%$ (Small N), 50 \% (Medium N), and 80 \% (Large N) to detect Cohens's d of 0.4 . 


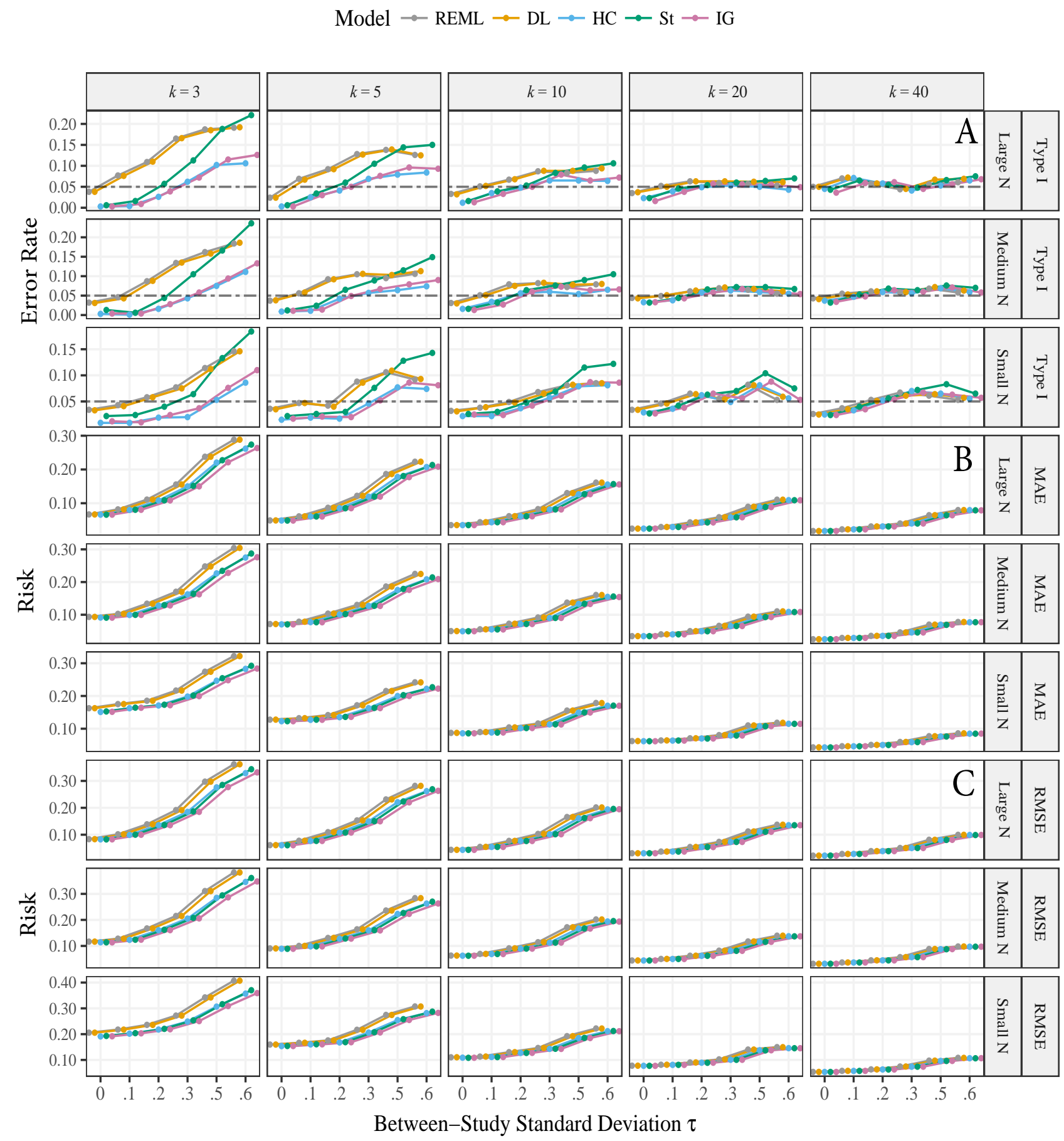

Figure 4. A: Type I error rates $(\mu=0)$. B: Mean absolute error. C: Root mean squared error. DL $=$ DerSimonian and Laird; REML $=$ Restricted Maximum Likelihood; HC = Half-Cauchy; St = Student-t; IG = Inverse-gamma. The total studies included in each meta-analysis is denoted with $k$. The sample sizes in the primary studies correspond to average power of $20 \%$ (Small N), $50 \%$ (Medium N), and $80 \%$ (Large N) to detect Cohens's d of 0.4 . 


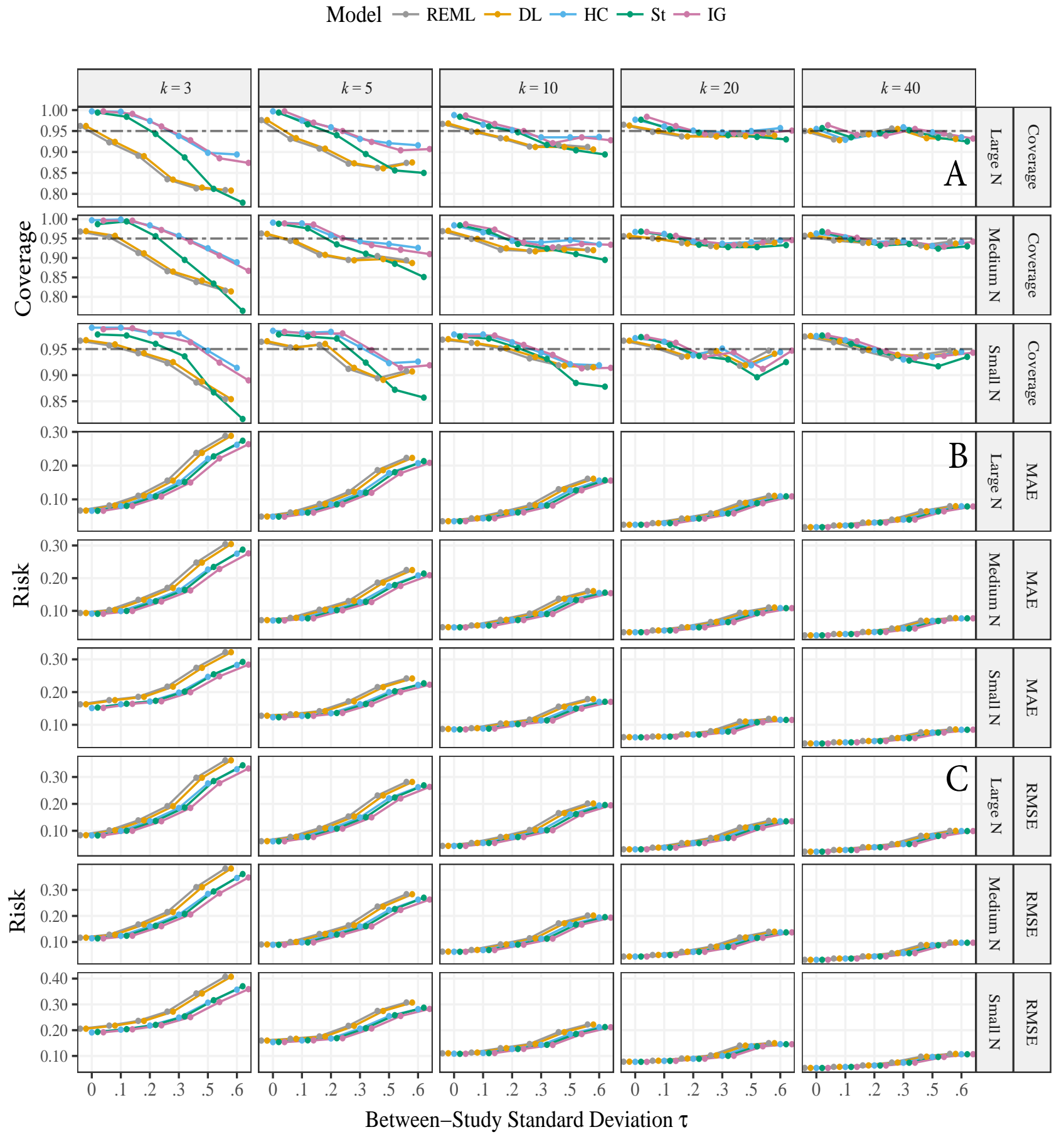

Figure 5. A: Coverage probabilities $(\mu=0.4)$. B: Mean absolute error. C: Root mean squared error. $D L=$ DerSimonian and Laird; $R E M L=$ Restricted Maximum Likelihood; HC = Half-Cauchy; St $=$ Student-t $; G=$ Inverse-gamma. The total studies included in each meta-analysis is denoted with $k$. The sample sizes in the primary studies correspond to average power of $20 \%$ (Small N), 50 $\%$ (Medium N), and $80 \%$ (Large N) to detect Cohens's d of 0.4 . 


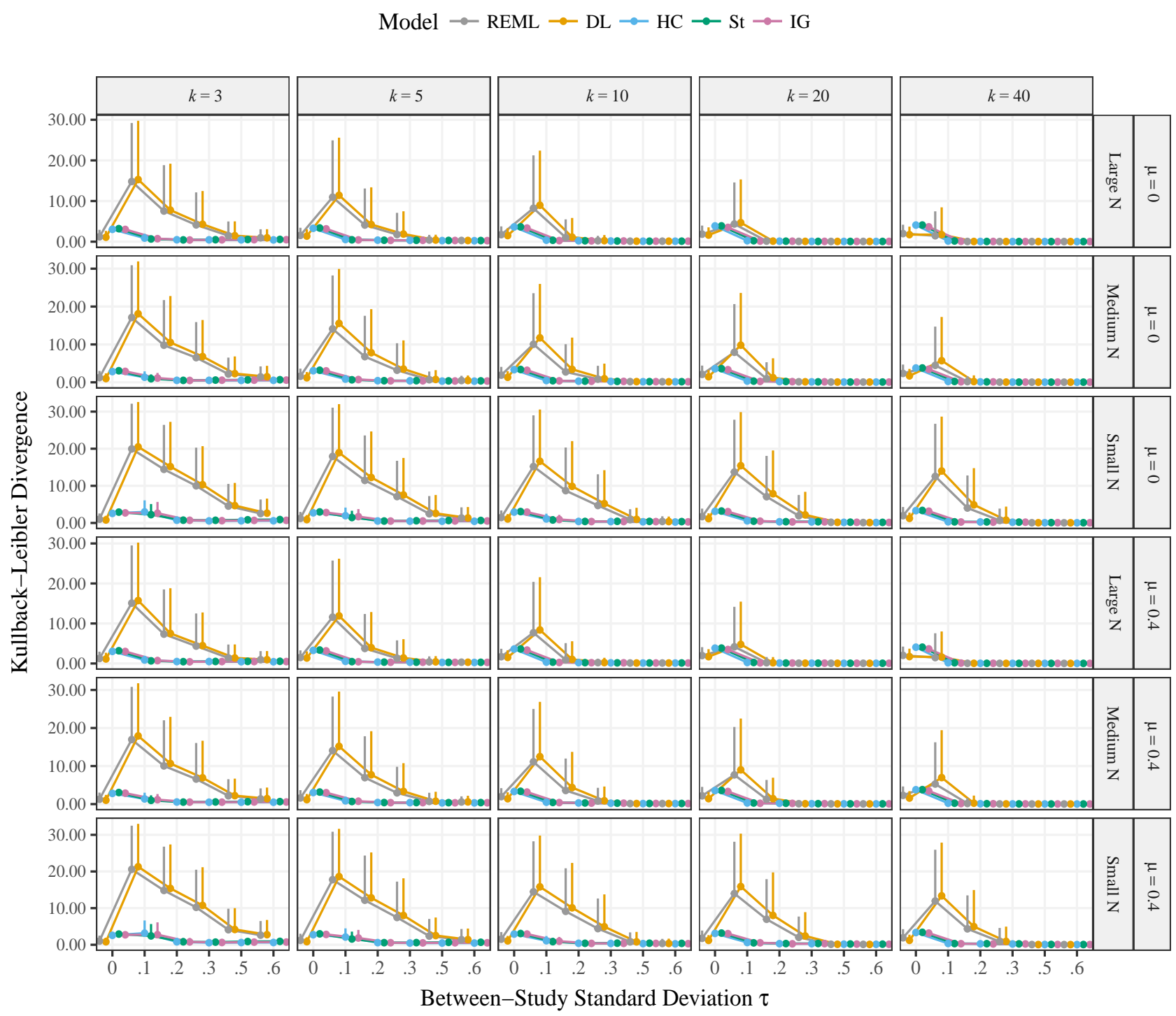

Figure 6. Kullback-Leibler Divergence (lower is closer to the true meta-analytic distribution) A: True null hypothesis $(\mu=0$ ). B: False null hypothesis $(\mu=0.4)$. DL = DerSimonian and Laird; REML = Restricted Maximum Likelihood; HC = Half-Cauchy; $S t=$ Student- $t ; G=$ Inverse-gamma. The total studies included in each meta-analysis is denoted with $k$. The sample sizes in the primary studies correspond to average power of $20 \%$ (Small N), 50 \% (Medium N), and $80 \%$ (Large N) to detect Cohens's d of 0.4 . Error bars represent the standard deviation.

is root mean squared error (RMSE) that is defined as

$$
\text { RMSE }=\sqrt{\frac{1}{n} \sum_{i=1}^{n}(\hat{\theta}-\theta)^{2}}, \quad \theta \in \Theta,
$$

where the risk is averaged across the $n$ simulation trials. This loss function was selected because it is most commonly used to evaluate estimators and predictions in many contexts. However, it has been noted that RMSE is sensitive to outliers (due considering the squared difference) and may not accurately reflect the true risk associated with an esti- mator (Berger, 2013). Therefore, we also computed mean absolute error (MAE) that is defined as

$$
\mathrm{MAE}=\frac{1}{n} \sum_{i=1}^{n}|\hat{\theta}-\theta|, \quad \theta \in \Theta .
$$

The results for the loss functions related to the estimation of $\tau$ are presented in Figure 3 (panels B and C). When $\tau$ was zero, the classical methods for estimating $\tau$ had lower risk, except for RMSE with $k$ of three. For positive values of $\tau$, however, the Bayesian methods consistently had lower risk 
when the number of studies in the meta-analyses were 3 and 5. This was the case for both RMSE and MAE. Interestingly, the errors were related to the sample sizes in the primary studies. That is, when the original studies had $80 \%$ power to detect $d$ of 0.40 , the risk was consistently lower than for underpowered studies. Furthermore, both RMSE and MAE increased with larger values of $\tau$, particularly when $k$ was small. In other words, when primary studies were more variable, there was greater risk associated with the estimates of $\tau$. With $k=3$ and $\tau=0.60$ in particular, RMSE indicated that the estimates had substantial variability. Importantly, for all models the risk diminished with larger values for $k$ and the models became similar to one another.

While the different priors were very similar, there were some notable differences. For example, with smaller values for $\tau$, the Student- $t$ prior often had the lowest risk, whereas higher risk was observed for larger values. As shown in Figure 1, this prior has light tails and a small scale, which in this case shrunk larger values towards zero and resulted in the distinct risk function. We also noted that the $\mathcal{H C}$ prior often had superior performance than $\mathcal{I G}$ for smaller values, whereas the opposite pattern was observed for larger values of $\tau$.

\section{Population Average}

Type 1 Error Rates. Type I error rates were computed as the proportion of simulation trials in which the confidence or highest density intervals of $\mu$ excluded zero. In other words, we evaluated coverage probabilities for $\mu=0$. The results are presented in Figure 4 (panel A), and reflect what was implied by the motivating examples (Figure 2). That is, the distribution of $p$-values showed opposing patterns between the classical and Bayesian models: the classical method had too many small $p$-values, whereas the Bayesian methods did not "have enough" small $p$-values to maintain the expected error rate $(\alpha=0.05)$. As shown in Figure 4, this resulted in substantial differences in terms of error rates. For the small $k$ values (3 and 5), for example, the error rates for the classical methods approached 0.20 , which was much larger than the error rates for the Bayesian models. Moreover, error rates were associated with the sample sizes in the primary studies. Also, as $k$ increased all models eventually converged to the expected error rate of 0.05 , irrespective of between-study variability.

All of the prior distributions lead again to very similar results, but there were still some notable differences to consider. The Student $-t^{+}$prior, for example, had the highest error rates of all models when $\tau$ was large. This was not surprising, because we expected the thin tails (Figure 1) to shrink larger values for $\tau$ which then produced overly confident (too narrow) probability densities. There were slight difference between $\mathcal{H C}$ and $\mathcal{I G}$, in that the latter had higher error for larger values of $\tau$. This highlights that the heavy tails of the half-Cauchy distribution are useful across a range of situations. When between-study variance was absent $(\tau=0)$, the classical methods were close to nominal levels ( $\alpha=0.05$ ), whereas the Bayesian methods almost always covered the population value. This suggests that uncertainty was overstated (the intervals were too wide), which may not be desirable when simply using null-hypothesis significance-testing for inference.

Coverage Probabilities. Here we assumed the null hypothesis was false, and examined coverage probabilities for a non-zero population value $(d=0.4)$. We expected similar performance as the type I error rates. Indeed, the results were so similar that we do not reiterate them here (note that 1 minus the type I error rate is coverage for the value $0)$. Nonetheless, the results are informative with respect to the prior we chose for the population value: $\mu \sim \mathcal{N}(0,1)$, which we considered as diffuse (at least for typical Cohen's $d$ values). The results presented in Figure 5 confirmed that this level of informativeness does not have much (if at all) influence on the estimates compared to when the true value was zero (Figure 4).

Loss Functions. We considered the same loss functions for estimating $\mu$ than we did for estimating $\tau^{2}$. The results are presented in Figures $4(\mu=0)$ and $5(\mu=0.4)$. Here the differences between classical and Bayesian methods were not as pronounced as for estimating $\tau$. For the smaller $k$ values (3, 5 , and 10), the Bayesian estimates had lower risk than the classical estimators for larger values of between-study variation. Furthermore, as $\tau$ increased, the differences between methods in terms of risk became larger.

These results also highlight general issues in estimating meta-analytic models with few studies. The estimates of the population averages had substantial variability according to RMSE, and the risk became even greater with more variability between-studies. Of course, with more studies included in the meta-analyses, both RMSE and MAE decreased to what may be considered more tolerable risk. Nonetheless, even for estimating the population average $\mu$, these results suggest that evaluating the corresponding risk function can provide valuable information.

\section{Kullback-Leibler Divergence}

The previous loss functions (RMSE and MAE) considered $\tau$ and $\mu$ separately. However, for a given model, both values are estimated at the same time. We thus considered Kullback-Leibler (KL) divergence-entropy loss-which measures divergence between probability distributions over the same variable (Kullback \& Leibler, 1951; McElreath, 2016). We computed the divergence between the estimated metaanalytic distribution

$$
p=\mathcal{N}(\hat{\mu}, \hat{\tau})
$$


and the true meta-analytic distribution

$$
q=\mathcal{N}(\mu, \tau)
$$

Here, $p$ and $q$ represent the corresponding densities of the distributions. When comparing continuous distributions, KL divergence follows

$$
\mathrm{KL}(p(x) \| q(x))=\int_{-\infty}^{\infty} p(x) \ln \frac{p(x)}{q(x)} d x
$$

where $\mathrm{KL}(p \| q)$ denotes divergence from $q$ to $p$. We then took the average KL-divergence across the simulation trials, where lower values indicate less discrepancy from the true meta-analytic distribution.

The results are presented in Figure 6. There were clear differences, in that the Bayesian methods consistently provided a better approximation to the true meta-analytic distribution than the classical methods. The degree of discrepancy was surprising, so we investigated what was responsible for the observed differences. We found that the divergence was larger and more variable due to boundary estimates or estimates very close to zero. Indeed, the observed pattern was very similar to that presented in Figure 6 (panel A). The Bayesian models had very similar performance across all conditions, such that for positive values $(\tau$ $>0$ ) the risk was consistent and generally lower than the classical methods. Importantly, for the largest $k$ value and sample sizes in the primary studies (Large $N$ ), the classical methods eventually achieved similar performance as the Bayesian models.

\section{Discussion}

In the present paper, we compared several classical and Bayesian meta-analytic random effects models with regard to their frequentist properties. Models mainly differed in the way the between study variance $\tau^{2}$ was estimated: We used two classical estimators (REML and DL) and three different priors in the Bayesian models. Overall, results indicate better performance of the Bayesian models both with respect to the estimation of the meta-analytic effect $\mu$ and the between study variance $\tau^{2}$. Possibly most striking were the differences in the latter parameter, which was mainly the result of Bayesian models not producing any boundary estimates $\left(\tau^{2}=0\right)$, while classical methods suffered heavily from this problem especially in case of very few studies. Importantly, the results demonstrated that estimating $\tau^{2}$ is not only relevant for its own purpose, but also influences estimation of the summary effect $\mu$ and the corresponding inferences.

It is also clear that summarizing evidence with few studies is a difficult task. However, as indicated by the motivating examples (Figure 2), this is often done in psychology and it is thus important to chose a method that minimizes risk.
In this regard, classical methods often provided less than nominal coverage of the summary effect $\mu$, thus overstating the certainty in the estimates. In contrast, Bayesian methods were more (sometimes too) conservative, but often had smaller risk for the quadratic and absolute loss functions. With respect to Kullback-Leibler divergence that considered $\mu$ and $\tau$ simultaneously, the Bayesian models had far superior performance (Figure 6 ). We should note that neither of the methods performed particularly well in case of small number of studies and large between-study heterogeneity, which is not uncommon psychological meta-analyses (van Erp et al., 2013). These results therefore suggest that, as a field, we might have for too long relied upon very few studies to make strong claims, without appreciating the risk associated with such inferences.

\section{Weakly-Informative Priors}

Within the Bayesian approach, we demonstrated the usefulness of weakly-informative priors (as compared to "non-informative" flat priors) (Gelman, 2006; Polson \& Scott, 2012). Such weakly-informative priors can be derived without much specific subject-matter-knowledge, but just by understanding the metric of the study effects and properties of probability distributions. For instance, in psychology, typical effect sizes such as Cohen's $d$ and Pearson's correlation $r$ are well understood by the vast majority of researchers. This allows for inferring an expected range for the summary effect. Based on this knowledge, specifying associated weakly-informative priors is not a very complicated task (Rcode provided in the Appendix). Through both our case studies and extensive simulations, we demonstrated that researchers do not need to be worried about making their analysis overly 'subjective' (and thus less credible) by adding weakly-informative priors. In fact, such priors are generally advised to guard against overfitting by restricting estimates to plausible values (McElreath, 2016). Notably, this view of prior distributions is analogous to penalized maximum likelihood (Cole, Chu, \& Greenland, 2014), so similar goals can be achieved with classical methods (e.g., LASSO), although not for variance components (to our knowledge) and without parameter uncertainty (Park \& Casella, 2008).

The applied weakly-informative half-Cauchy prior and the informed inverse-Gamma prior led to very similar posterior estimates, despite being somewhat different (Figure 1). This similarity in performance is important, because it demonstrates that informative priors do not need to be based on empirical data to achieve improved performance. In contrast, the more narrow half-t-prior led to an underestimation of $\tau^{2}$ in case of high between study heterogeneity. This is of course not surprising, as this prior puts not enough weight on larger values of $\tau^{2}$, but it nicely demonstrates that using priors with small tails can lead to an underestimation of parameters. Thus, when specifying appropriate weakly- 
informative priors, one has to balance between penalizing (putting few prior weight on) large and unrealistic parameter values, while still allowing larger values to be possible if the data suggests they are reasonable (Gelman et al., 2017).

\section{Frequentist Properties of Bayesian Models}

Bayesian methods are often promoted in stark contrast to classical methods (Kruschke, 2013). Indeed, some authors explicitly state that inferential goals differ between Bayesian and frequentist approaches. We agree, but a distinction must be made between investigating frequentist properties of our models and making frequentist inferences. Examining properties under repeated use has a long tradition (Rubin, 1984) and is the dominant approach to Bayesian statistics (Berger, 2006). In simulation, we know the truth and random sampling allows for investigating contexts under which a proposed method has suboptimal performance (Gelman, 2011). For example, our results demonstrated that the posterior probability density of the summary effect was influenced by between-study variation. We view examining our models' expected performance as extremely useful for evaluating statistical models and Bayesian evidential quantities (Rubin, 1984). However, hypothetical data frequencies should inform rather than determine statistical practice (Greenland, 2017), such as guiding the selection of a method based on risk minimization.

Further, there are other concepts such as type S (sign) and M (magnitude) errors (Gelman \& Carlin, 2014; Gelman \& Tuerlinckx, 2000), which are arguably more appropriate to describe errors in statistical inference than type I and II errors (Chen et al., 2017; Gelman, Hill, \& Yajima, 2012). For the present paper, we still went with the latter as the most common and well understood approach to not only allow for comparisons with other simulation studies, but also to reach a wider audience. Finally, all statistics have frequency properties (Hoijtink, van Kooten, \& Hulsker, 2016), whereas not all statistics have probabilities for evaluation. That is, it is not possible to assess classical methods in terms of Bayesian assumptions (e.g., parameters as random variables) (Andradóttir \& Bier, 2000) such as posterior quantile (Cook, Gelman, \& Rubin, 2006) and prior predictive calibration (Garc \& Chen, 2005) as well as Bayesian risk (Berger, 2013). Bayesian methods are more general; for example, measures of central tendency for frequency evaluation minimize a specific Bayesian loss function (mean = squared error; median $=$ absolute error; mode $=0-1$ loss function) across the posterior probability distribution, whereas only a point estimate is provided by classical estimators and thus require repeated sampling to assess risk (Robert, 2007).

\section{Limitations}

There are several potential limitations to consider. First, we investigated only random effects but no fixed effects models in our simulations, although the latter constituted the true model for some simulation conditions. This was done because (a) between study heterogeneity is the norm rather than the exception in psychology (van Erp et al., 2013) and we thus focused on the model class with far more practical relevance; (b) random effects models are a generalization of fixed effects models and will thus reduce to fixed effects model if enough evidence is provided (Schmidt et al., 2009); and (c) the random effects model allows for unconditional inferences, in which inferences are not restricted to the observed studies (Hedges \& Vevea, 1998). Second, we did not consider statistical tests of the "significance" of $\tau^{2}$ to choose between fixed and random effects models. Although common in practice, these tests have undesirable statistical properties (Hardy \& Thompson, 1998) and are from our perspective not advised to determine the most appropriate meta-analytic model (Viechtbauer, 2010). Further, the desired inference should guide which model to use and not a null hypothesis significance test (Hedges \& Vevea, 1998). Third, there are many reasonable (families of) prior distributions to consider. Here we focused only on two families of which we choose three particular priors. They serve as illustrative examples and are not meant to be representative for the whole set of options in this regard. Further, the scale of the applied priors is not necessarily reasonable for effects sizes other than Cohen's $d$, and this should be investigated going forward. Fourth, we did not consider a so-called uninformative prior (e.g. uniform), which may be viewed as a limitation. The decision was made because, in several studies, it was shown that these priors should not be used for variance components (Depaoli \& Clifton, 2015; Polson \& Scott, 2012), in addition to being unjustifiable based on previous information about $\tau$ in psychology (van Erp et al., 2013).

\section{Guidelines for Applied Researchers}

Although uncommon in psychology, researchers can view choosing a statistical model as an explicit decision. The figures in this work represent risk functions and a model can then be selected based on risk minimization of a particular loss function (Berger, 2013). Given strong theoretical justification (McElreath, 2016; Shlens, 2014), we would find it difficult to chose a loss function other than KL-divergence, but this is of course a question specific consideration that can be informed by Figure 6. In this light, if we restrict our choice to these simulation conditions and methods, there is a clear justification for using Bayesian meta-analytic methods with weakly-informative priors that does not depend on critiquing $p$-values or arguing that Bayesian methods are inherently superior.

We also encourage researchers to not rely exclusively on statistical estimates, but also to think about whether they make sense for their particular research question, especially 
in the context of relevant information. Notions such as unbiasedness depend on fitting the true model (e.g., as in these simulations), in which bias is assessed from a user defined population. Without consideration of relevant information, this can result in misleading inferences and may conflict with common observations (Clarke, 2009; Harris \& Robinson, 2007). Researchers can consider whether zero variance (e.g., a boundary estimate) makes sense, or is a realistic expectation, for example, rather than rely exclusively on a statistical model to make this determination. Indeed, some have argued that zero between-study variance is untenable (Brockwell \& Gordon, 2001).

For Bayesian meta-analysis, we would avoid adopting the 0 - 1 loss function implicit to null hypothesis significance testing (Robert, 2007). Of course, as the present results demonstrate, researchers can also make frequentist inferences from Bayesian models. However, the estimated posterior distributions can be interpreted probabilistically as plausible values for the true effect (Greenland \& Poole, 2013; Harrell \& Shih, 2001), thus avoiding categorizing continuous evidence into significant vs. non-significant (Amrhein \& Greenland, 2017; Mcshane, Gal, Gelman, Robert, \& Tackett, 2017). This can be accomplished with highest density intervals, in addition to posterior probabilities in the predicted direction (e.g., $p(\mu>0 \mid y, \mathcal{M}))$. Importantly, while these probabilities are conditional on the observed data and all other model assumptions (e.g., the prior distribution), they overcome conditioning on the truth being zero. Furthermore, sensitivity analyses should be conducted to see how evidence changes by varying reasonable prior distributions, especially for the between-study variance (Carlsson et al., 2017). This can be done to not only demonstrate robustness, but also as an attempt to provide more cautious conclusions. Finally, since inference can be viewed as what would follow from the assumptions, all must be clearly articulated and justified (Depaoli \& van de Schoot, 2017). This does not only apply to the prior distribution, but also to other assumptions such as the likelihood (e.g., a normal distribution for observed effect sizes).

We have provided annotated R-code in the Appendix to fit Bayesian meta-analytic models with the R package brms (Bürkner, 2017), which is based on Stan (Stan Development Team, 2015) and thus uses the same engine as the Bayesian models in our simulations.

\section{Appendix \\ R-code}

1) install Stan: windows install and mac or linux install

2) install brms: brms installation

3) install metaBMA metaBMA installation

4) install metafor metafor installation

library(brms)

library(metaBMA)

library(metafor) \# to compare code needed to fit the Bayesian model stein, $\mathrm{H}$. R.

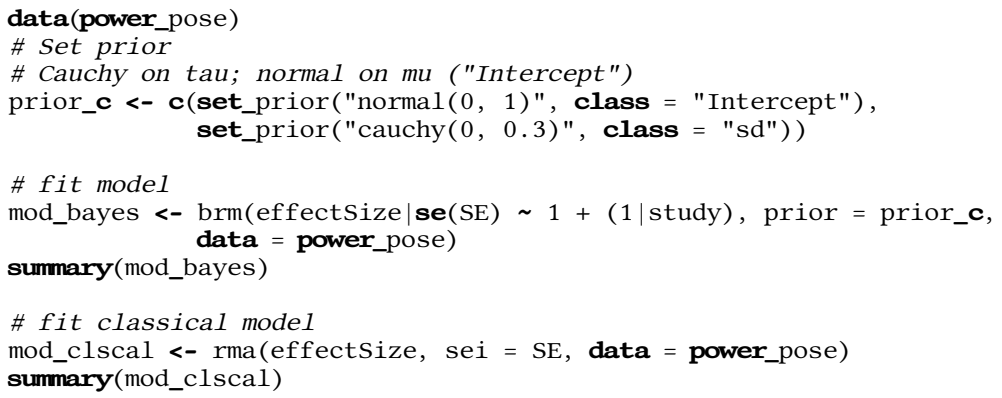

We refer readers to Depaoli and van de Schoot (2017) for a checklist of items to ensure the Bayesian model makes sense (e.g., chain convergence) and can be reproduced.

\section{References}

Amrhein, V., \& Greenland, S. (2017). Remove, rather than redefine, statistical significance. Nature Human Behaviour, 133(2016), 1. Retrieved from http://www. nature .com/articles/s41562-017-0224-0 doi: 10 .1038/s41562-017-0224-0

Andradóttir, S., \& Bier, V. M. (2000). Applying Bayesian ideas in simulation. Simulation Practice and Theory, 8(3-4), 253-280. doi: 10.1016/S0928-4869(00)00025-2

Asparouhov, T., \& Muthén, B. (2010). Bayesian analysis of latent variables models using Mplus.

Baldwin, D. S., Anderson, I. M., Nutt, D. J., Bandelow, B., Bond, A., Davidson, J. R. T., ... Scott, J. (2005). Evidence-based guidelines for the pharmacological treatment of anxiety disorders: recommendations from the British Association for Psychopharmacology. Journal of Pharmacology, 19(6), 567596. doi: $10.1177 / 0269881110391123$

Bates, D., Mächler, M., Bolker, B. M., \& Walker, S. C. (2014, 6). Fitting linear mixed-effects models using lme4. arXiv:1406.5823v1[stat.CO]23, 1 - 51 . Retrieved from http://arxiv.org/abs/1406.5823 doi: 10 $.1177 / 009286150103500418$

Berger, J. (2003). Could Fisher, Jeffreys and Neyman Have Agreed on Testing? Statistical Science, 18(1), 1-32. Retrieved from http://projecteuclid .org/euclid.ss/1056397485 doi: 10.1214/ss/ 1056397485

Berger, J. (2006). The case for objective Bayesian analysis. Bayesian Analysis, 1(3), 385-402. Retrieved from https://www2.stat.duke.edu/ berger/papers/obayes-debate.pdf doi: 10.1214/06-BA115

Berger, J. (2013). Statistical decision theory and Bayesian analysis. Springer Science \& Business Media.

Betancourt, M. (2017, 1). A Conceptual Introduction to Hamiltonian Monte Carlo. Retrieved from http://arxiv . org/abs/1701.02434

Borenstein, M., Hedges, L., Higgins, J., \& Rothstein, H. R. (2005). Comprehensive meta-analysis version 2. Englewood: Biostat.

Borenstein, M., Hedges, L. V., Higgins, J. P. T., \& Roth(2009). Introduction to Meta-Analysis. 
Retrieved from http://doi.wiley.com/ 10.1002/9780470743386. fmatter doi: 10.1002/9780470743386.fmatter

Brockwell, S. E., \& Gordon, I. R. (2001). A comparison of statistical methods for meta-analysis. Statistics in medicine, 20(6), 825840. doi: 10.1002/sim.650

Bürkner, P.-C. (2017). brms : An R Package for Bayesian Multilevel Models Using Stan. Journal of Statistical Software, 80(1). Retrieved from http: //www. jstat soft.org/v80/ i01/ doi: 10.18637/jss.v080.i01

Bürkner, P.-C., Williams, D., Simmons, T., \& Woolley, J. (2017). Intranasal Oxytocin May Improve High-Level Social Cognition in Schizophrenia, but Not Social Cognition or Neurocognition in General: A Multilevel Bayesian Metaanalysis. Schizophrenia Bulletin, 43(6). doi: 10.1093/schbul/ sbx053

Carlsson, R., Schimmack, U., Williams, D., \& Bürkner, P.-C. (2017). Bayes Factors From Pooled Data Are No Substitute for Bayesian Meta-Analysis: Commentary on Scheibehenne, Jamil, and Wagenmakers (2016). Psychological Science, 28(11). doi: 10.1177/0956797616684682

Chen, G., Xiao, Y., Taylor, P. A., Riggins, T., Geng, F., \& Redcay, E. (2017). Handling Multiplicity in Neuroimaging through Bayesian Lenses with Hierarchical Modeling. , 1-32. doi: $10.1101 / 238998$

Chung, Y., Rabe-Hesketh, S., \& Choi, I. H. (2013). Avoiding zero between-study variance estimates in random-effects metaanalysis. Statistics in Medicine, 32(23), 4071-4089. doi: 10.1002/sim.5821

Chung, Y., Rabe-Hesketh, S., Dorie, V., Gelman, A., \& Liu, J. $\quad(2013,10) . \quad$ A Nondegenerate Penalized Likelihood Estimator for Variance Parameters in Multilevel Models. Psychometrika, 78(4), 685-709. Retrieved from http://1ink.springer.com/ 10.1007/s11336-013-9328-2 doi: 10.1007/ s11336-013-9328-2

Clarke, K. A. (2009). Return of the phantom menace: Omitted variable bias in political research. Conflict Management and Peace Science, 26(1), 46-66. doi: 10.1177/0738894208097666

Cole, S. R., Chu, H., \& Greenland, S. (2014). Maximum likelihood, profile likelihood, and penalized likelihood: A primer. American Journal of Epidemiology, 179(2), 252-260. doi: 10.1093/aje/kwt245

Cook, S. R., Gelman, A., \& Rubin, D. B. (2006). Validation of software for Bayesian models using posterior quantiles. Fournal of Computational and Graphical Statistics, 15(3), 675-692. doi: 10.1198/106186006X136976

Curran, P. J., \& Hussong, A. M. (2009). Integrative Data Analysis: The Simultaneous Analysis of Multiple Data Sets. Psychological Methods, 14(2), 81-100. doi: 10.1037/a0015914

Depaoli, S., \& Clifton, J. P. (2015). A Bayesian Approach to Multilevel Structural Equation Modeling With Continuous and Dichotomous Outcomes. Structural Equation Modeling, 22(3), 327-351. Retrieved from http : / / dx . doi.org/ $10.1080 / 10705511.2014 .937849$ https : / / doi.org/10.1080/10705511.2014.937849 doi: 10.1080/10705511.2014.937849

Depaoli, S., \& van de Schoot, R. (2017). Improving trans- parency and replication in Bayesian statistics: The WAMBSchecklist. Psychological Methods, 22(2), 240-261. Retrieved from http://doi.apa.org/getdoi.cfm?doi= $10.1037 /$ met 0000065 doi: $10.1037 /$ met0000065

Dersimonian, R., \& Laird, N. (1986). Meta-Analysis in Clinical Trials. Statistics in medicine, 188, 177-188. doi: 10.1016/ 0197-2456(86)90046-2

Friede, T., Röver, C., Wandel, S., \& Neuenschwander, B. (2017). Meta-analysis of few small studies in orphan diseases. Research Synthesis Methods, 8(1), 79-91. doi: 10.1002/jrsm .1217

Garc, G., \& Chen, M.-h. (2005). Calibrating Bayes Factor Under Prior Predictive Distributions. , 15, 359-380.

Garrison, K. E., Finley, A. J., \& Schmeichel, B. J. (2017). Ego Depletion Reduces Attention Control: Evidence from Two HighPowered Preregistered Experiments. PsyArXiv. Retrieved from https://www.tandfonline.com/doi/ full/10.1080/23743603.2017.1326760 doi: 10.17605/OSF.IO/PGNY3

Gelman, A. (2006). Prior distributions for variance parameters in hierarchical models (Comment on Article by Browne and Draper). Bayesian Analysis, 1(3), 515-534. doi: 10.1214/ 06-BA117A

Gelman, A. (2011). Bayesian Statistical Pragmatism. Statistical Science, 26(1), 10-11. Retrieved from http: //www. stat. columbia.edu/ gelman/ research/published/kassdiscussion.pdf doi: $10.1214 / 10-S T S 337$

Gelman, A. $(2013,1)$. Commentary: P Values and Statistical Practice. Epidemiology, 24(1), 69-72. Retrieved from http://ovidsp.tx.ovid . com/sp-3.8.0b/ovidweb.cgi?\&S=

KLEOFPAFEBDDHOFNNCOKCGOBCHMAAA0 0\&Link+ Set $=$ S. sh $.18 .19 .22 .25 \% 7 C 10 \% 7 C s 1_{-} 10$ doi: 10.1097/EDE.0b013e31827886f7

Gelman, A., \& Carlin, J. (2014). Beyond Power Calculations: Assessing Type S (Sign) and Type M (Magnitude) Errors. Perspectives on Psychological Science, 9(6), 641-651. doi: 10.1177/1745691614551642

Gelman, A., Carlin, J. B., Stern, H. S., Dunson, B. D., Vehtari, A., \& Rubin, D. B. (2014). Bayesian Data Analysis. Boca Raton: CRC Press. Retrieved from https : //books.google.com/books/about/ Bayesian_Data_Analysis_Third_Edition .html?id=ZXL6AQAAQBAJ

Gelman, A., \& Dyk, D. a. V. (2007). Transformed and parameterexpanded Gibbs samplers for multilevel linear and generalized linear models. Elements, 1-31.

Gelman, A., Hill, J., \& Yajima, M. (2012, 4). Why We (Usually) Don't Have to Worry About Multiple Comparisons. Fournal of Research on Educational Effectiveness, 5(2), 189-211. Retrieved from http://www.tandfonline.com/ doi/abs/10 . 1080/19345747.2011.618213 doi: $10.1080 / 19345747.2011 .618213$

Gelman, A., Jakulin, A., Pittau, M. G., \& Su, Y. S. (2008). A weakly informative default prior distribution for logistic and other regression models. Annals of Applied Statistics, 2(4), 13601383. doi: 10.1214/08-AOAS191 
Gelman, A., Simpson, D., \& Betancourt, M. (2017). The prior can generally only be understood in the context of the likelihood. ArXiv preprint. Retrieved from http://arxiv . org/abs/1708.07487

Gelman, A., \& Tuerlinckx, F. (2000, 9). Type S error rates for classical and Bayesian single and multiple comparison procedures. Computational Statistics, 15(3), 373-390. Retrieved from http://1ink.springer.com/10 .1007 /s001800000040 doi: 10.1007/s001800000040

Goldstein, M. (2006). Subjective Bayesian Analysis : Principles and Practice Applied subjectivism. Bayesian Analysis, 1(3), 403-420.

Greenland, S. (2017). Invited Commentary: The Need for Cognitive Science in Methodology. American fournal of Epidemiology, 186(6), 639-645. doi: 10.1093/aje/kwx259

Greenland, S., \& Poole, C. $(2013,1)$ Living with $\mathrm{P}$ Values. Epidemiology, 24(1), 62-68. Retrieved from http://content.wkhealth . com/1inkback/openurl?sid=WKPTLP :

landingpage\&an $=00001648-201301000$ - 00009\%5Cnpapers $2: / / p u b 1$ ication/doi/ 10.1097/EDE.0b013e3182785741 doi 10.1097/EDE.0b013e3182785741

Gronau, Q. F., Van Erp, S., Heck, D. W., Cesario, J., Jonas, K. J., \& Wagenmakers, E.-J. (2017). A Bayesian model-averaged meta-analysis of the power pose effect with informed and default priors: the case of felt power. Comprehensive Results in Social Psychology, 2(1), 123-138. Retrieved from https://www.tandfonline.com/doi/ $\mathrm{full} / 10.1080 / 23743603.2017 .1326760$ doi: $10.1080 / 23743603.2017 .1326760$

Guolo, A., \& Varin, C. (2017). Random-effects meta-analysis: The number of studies matters. Statistical Methods in Medical Research, 26(3), 1500-1518. doi: 10.1177/0962280215583568

Haidich, A. B. $(2010,12)$ Meta-analysis in medical research. Hippokratia, 14(Suppl 1), 29-37. Retrieved from http://www.pubmedcentral .nih.gov/articlerender.fcgi?artid= 3049418\&tool $=$ pmcent rez\&rendertype $=$ abstract doi: 10.5005/jp/books/10519

Hardy, R. J., \& Thompson, S. G. (1998). Detecting and describing heterogeneity in meta-analysis. Statistics in Medicine, 17(8), 841-856. doi: 10.1002/(SICI)1097-0258(19980430)17:8<841:: AID-SIM781>3.0.CO;2-D

Harrell, F. E., \& Shih, Y. C. T. (2001). Using full probability models to compute probabilities of actual interest to decision makers. International journal of technology assessment in health care, 17(1), 17-26. doi: 10.1017/S0266462301104034

Harris, A. L., \& Robinson, K. (2007). Schooling Behaviors or Prior Skills ? , 80, 139-157.

Heck, D. W., Gronau, Q. F., \& Wagenmakers, E.-J. (2017). metaBMA: Bayesian Model Averaging for Random and Fixed Effects Meta-Analysis. Retrieved from https : / / cran.r - project . org / package =metaBMA

Hedges, L. V., \& Vevea, J. L. (1998). Fixed- and random-effects models in meta-analysis. Psychological Methods, 3(4), 486-504. Retrieved from http://personal.psc . isr.umich . edu/yuxie-web/files/pubs/
Articles/Hedges_Vevea1998.pdfhttp://

doi.apa.org/getdoi.cfm?doi=10.1037/

1082-989X. 3.4. 486 doi: 10.1037/1082-989X.3.4.486

Higgins, J., \& Spiegelhalter, D. (2002). Being sceptical about metaanalyses: a Bayesian perspective on magnesium trials in myocardial infarction. International journal of epidemiology, 31(1), 96-104. doi: 10.1093/ije/31.1.96

Higgins, J., \& Thompson, S. (2002). Quantifying heterogeneity in meta - analysis. Statistics in Medicine, 21(11), 1539-1558. Retrieved from http: //onlinelibrary .wiley.com/doi/10.1002/sim.1186/full

Hoffman, M., \& Gelman, A. (2014). The No-U-Turn Sampler: Adaptively Setting Path Lengths in Hamiltonian Monte Carlo. Journal of Machine Learning Research, 15(1), 30.

Hoijtink, H., van Kooten, P., \& Hulsker, K. (2016). Bayes Factors Have Frequency Properties-This Should Not Be Ignored: A Rejoinder to Morey, Wagenmakers, and Rouder. Multivariate Behavioral Research, 51(1), 20-22. Retrieved from http: //dx.doi.org/10.1080/00273171 .2015.1071705 doi: 10.1080/00273171.2015.1071705

Hunter, J. E., \& Schmidt, F. L. (2000). Fixed Effects vs. Random Effects Meta-Analysis Models: Implications for Cumulative Research Knowledge. International fournal of Selection and Assessment, 8(4), 275-292. Retrieved from https://Www.biz.uiowa.edu/ faculty/fschmidt/meta-analysis/ Hunter_Schmidt_2000_rev.pdfhttp:// doi.wiley.com/10.1111/1468-2389.00156 doi: $10.1111 / 1468-2389.00156$

Hunter, J. E., \& Schmidt, F. L. (2004). Methods of Meta-Analysis: Correcting Error and Bias in Research Findings. Thousand Oaks: SAGE Publications.

Kenny, D. A., \& Judd, C. M. (2017). The Unappreciated Heterogeneity of Effect Sizes: Implications for Power, Precision, Planning of Research, and Replication. Retrieved from http : //davidakenny.net/doc/KJ17R.pdf

Klein, R. A., Ratliff, K. A., Vianello, M., Adams, R. B., Stepan, B., Bernstein, M. J., ... Nosek, B. A. (2014, 5). Investigating variation in replicability: A "many labs" replication project. Social Psychology, 45(3), 142-152. Retrieved from http : / / econtent.hogrefe.com/ doi/abs/10.1027/1864-9335/a000178 doi: $10.1027 / 1864-9335 / \mathrm{a} 000178$

Kolenikov, S., \& Bollen, K. A. (2012). Testing Negative Error Variances: Is a Heywood Case a Symptom of Misspecification? (Vol. 40) (No. 1). doi: 10.1177/0049124112442138

Kruschke, J. K. (2013). Bayesian estimation supersedes the $\mathrm{t}$ test. Fournal of Experimental Psychology: General, 142(2), 573-603. Retrieved from http://doi.apa.org/ getdoi.cfm?doi=10.1037/a0029146 doi: 10 $.1037 / \mathrm{a} 0029146$

Kullback, S., \& Leibler, R. A. (1951). On information and sufficiency. The Annals of Mathematical Statistics. Retrieved from http://www.jstor.org/ stable/10.2307/2236703\%5Cnpapers2:// publication/uuid/A65B3271-44DC-42DC -87B5-A0C3A91EBFA8

Lambert, P. C., Sutton, A. J., Burton, P. R., Abrams, K. R., \& Jones, 
D. R. (2005). How vague is vague? A simulation study of the impact of the use of vague prior distributions in MCMC using WinBUGS. Statistics in Medicine, 24(15), 2401-2428. doi: $10.1002 / \operatorname{sim} .2112$

McElreath, R. (2016). Statistical Rethinking: A Bayesian Course with Examples in $R$ and Stan. CRC Press. Retrieved from https://books.google.com/books?hl= en\& $1 \mathrm{r}=\& \mathrm{i} d=\mathrm{mDo} 0 \mathrm{C}$ WAQBAJ $\& \mathrm{pg}$ is $=1$ doi: $10.3102 /$ 1076998616659752

McNeish, D. (2016). On Using Bayesian Methods to Address Small Sample Problems. Structural Equation Modeling, 23(5), 750773. doi: 10.1080/10705511.2016.1186549

Mcshane, B. B., Gal, D., Gelman, A., Robert, C., \& Tackett, J. L. (2017). Abandon Statistical Significance. , 1-12.

Meredith, M., \& Kruschke, J. (2016). HDInterval: Highest (Posterior) Density Intervals. Retrieved from https : / / cran . r-project . org / package $=$ HDInterval

Monnahan, C. C., Thorson, J. T., \& Branch, T. A. (2017). Faster estimation of Bayesian models in ecology using Hamiltonian Monte Carlo. Methods in Ecology and Evolution, 8(3), 339348. doi: 10.1111/2041-210X.12681

Overton, R. C. (1998). A Comparison of Fixed-Effects and Mixed (Random-Effects) Models for Meta-Analysis Tests of Moderator Variable Effects. Psychological Methods, 3(3), 354379. Retrieved from http : / / doi . apa . org/getdoi . cfm?doi=10.1037/1082-989X.3.3.354 doi: 10.1037/1082-989X.3.3.354

O’Donnell, M., Nelson, L. D., Ackermann, E., Aczel, B., Akhtar, A., Aldrovandi, S., ... Bialobrzeska, O. (2017). Registered Replication Report : Dijksterhuis \& van Knippenberg ( 1998 ) Multilab direct replication of : A variant of study 4 from Dijksterhuis , A . \& van Knippenberg , A . ( 1998 ). The relation between perception and behavior, or how to win a game of . , 1-38.

Park, T., \& Casella, G. (2008). The Bayesian Lasso. Fournal of the American Statistical Association, 103(482), 681-686. doi: 10.1198/016214508000000337

Polson, N. G., \& Scott, J. G. (2012). On the half-cauchy prior for a global scale parameter. Bayesian Analysis, 7(4), 887-902. doi: 10.1214/12-BA730

Pullenayegum, E. M. (2011). An informed reference prior for between-study heterogeneity in meta-analyses of binary outcomes. Statistics in Medicine, 30(26), 3082-3094. doi: $10.1002 / \operatorname{sim} .4326$

Raudenbush, S. W. (2009). Analyzing effect sizes: Random effects models. In H. Cooper, L. V. Hedges, \& J. C. Valentine (Eds.), The handbook of research synthesis and meta-analysis (2nd ed., pp. 295-315). New York: Russell Sage Foundation.

Raudenbush, S. W., \& Bryk, A. S. (2002). Hierarchical Linear Models: Applications and Data Analysis Methods (2nd ed.). Thousand Oaks: Sage Publications.

Rhodes, K. M., Turner, R. M., \& Higgins, J. P. T. (2015). Predictive distributions were developed for the extent of heterogeneity in meta-analyses of continuous outcome data. Journal of Clinical Epidemiology, 68(1), 52-60. Retrieved from http://dx.doi.org/10.1016/j .jclinepi.2014.08.012 doi: 10.1016/j.jclinepi .2014 .08 .012
Rhodes, K. M., Turner, R. M., White, I. R., Jackson, D., Spiegelhalter, D. J., \& Higgins, J. P. (2016). Implementing informative priors for heterogeneity in meta-analysis using metaregression and pseudo data. Statistics in Medicine, 35(29), 5495-5511. doi: 10.1002/sim.7090

Rice, K., Higgins, J. P. T., \& Lumley, T. (2017). A re-evaluation of fixed effect(s) meta-analysis. Fournal of the Royal Statistical Society. Series A: Statistics in Society, 205-227. doi: $10.1111 /$ rssa. 12275

Robert, C. (2007). The Bayesian choice: from decision-theoretic foundations to computational implementation. , 77 - 81.

Rubin, D. B. (1984). Bayesianly Justifiable and Relevant Frequency Calculations for the Applied Statistician. The Annals of Statistics, 12(4), 1151-1172. Retrieved from http : //projecteuclid.org/euclid.aos / 1176346785 doi: $10.1214 /$ aos/1176346785

Scheibehenne, B., Jamil, T., \& Wagenmakers, E.-J. (2016, 7). Bayesian Evidence Synthesis Can Reconcile Seemingly Inconsistent Results. Psychological Science, 27(7), 1043-1046. Retrieved from http: / / journals.sagepub.com/ doi/10.1177/0956797616644081 doi: 10.1177/ 0956797616644081

Schmidt, F. (1992). What do data really mean? Research findings, meta-analysis, and cumulative knowledge in psychology. American Psychologist, 47(10), 1173-1181. Retrieved from http: //doi.apa.org/getdoi.cfm?doi= 10.1037/0003-066X.47.10.1173 doi: 10.1037/ 0003-066X.47.10.1173

Schmidt, F. (2008). Meta-analysis: A constantly evolving research integration tool. Organizational Research Methods, 11(1), 96113. doi: $10.1177 / 1094428107303161$

Schmidt, F., Oh, I., \& Hayes, T. (2009, 2). Fixed- versus random-effects models in meta-analysis: Model properties and an empirical comparison of differences in results (Vol. 62) (No. 1). Blackwell Publishing Ltd. Retrieved from http://doi.wiley.com/10.1348/ $000711007 X 255327$ doi: 10.1348/000711007X255327

Shlens, J. (2014). Notes on Kullback-Leibler Divergence and Likelihood., 1-4. Retrieved from http://arxiv.org/ abs $/ 1404.2000$

Sidik, K., \& Jonkman, J. N. (2005). Simple heterogeneity variance estimation for meta-analysis. Journal of the Royal Statistical Society: Series C: Applied Statistics, 367-384.

Sidik, K., \& Jonkman, J. N. (2007, 4). A comparison of heterogeneity variance estimators in combining results of studies. Statistics in Medicine, 26(9), 1964-1981. Retrieved from ht $\mathrm{t} \mathrm{p}$ : / / doi.wiley.com/10.1002/sim.2688 doi: 10 $.1002 / \operatorname{sim} .2688$

Stan Development Team. (2015). \$1\$pkg\{\{\}Stan \{\}$\}: A$ $\$ 1$ proglang $\{\{\} C++\{\}\}$ Library for Probability and Sampling, Version 2.8.0. Retrieved from htt $\mathrm{p}: / / \mathrm{mc}-\mathrm{stan}$. org/

Sutton, A. J., Abrams, K. R., \& Health, P. (2001). Bayesian methods in meta-analysis and evidence synthesis. Statistical methods in medical research, 2802(01), 277-303. doi: 10.1191/ 096228001678227794

Turner, R. M., Davey, J., Clarke, M. J., Thompson, S. G., \& Higgins, J. P. $(2012$, 6). Predicting the extent of heterogeneity in meta-analysis, using empirical data 
from the Cochrane Database of Systematic Reviews. International fournal of Epidemiology, 41(3), 818-827. Retrieved from https://academic.oup.com/ije/ article-lookup/doi/10.1093/ije/dys041 doi: 10.1093/ije/dys041

Van Aert, R. C., \& Van Assen, M. A. (2017, 4). Bayesian evaluation of effect size after replicating an original study. PLoS ONE, 12(4), e0175302. Retrieved from http : / / dx . plos .org/10.1371/journal. pone.0175302 doi: 10.1371/journal.pone.0175302

van Erp, S., Verhagen, J., Grasman, R., \& Wagenmakers, E. (2013). Estimates of Between-Study Heterogeneity for 705 Meta-Analyses Reported in Psychological Bulletin From 1990-2013. (1), 1-18. Retrieved from https://www.gwern.net/docs/ statistics/2015-franco.pdf\%0Ahttp : / / journals.sagepub.com/doi/10.1177/ 1948550615598377

Veroniki, A. A., Jackson, D., Viechtbauer, W., Bender, R., Bowden, J., Knapp, G., ... Salanti, G. (2016, 3). Methods to estimate the between-study variance and its uncertainty in meta-analysis. Research
Synthesis Methods, 7(1), 55-79. Retrieved from http://www.ncbi.nlm.nih.gov/pubmed/ 26332144 http : / /www pubmedcentral .nih.gov/articlerender.fcgi?artid= PMC4950030 doi: 10.1002/jrsm.1164

Viechtbauer, W. (2007). Confidence intervals for the amount of heterogeneity in meta-analysis. Statistics in Medicine, 26(1), 37-52. doi: 10.1002/sim.2514

Viechtbauer, W. (2010). Conducting Meta-Analyses in $<\mathrm{i}>\mathrm{R}</ \mathrm{i}>$ with the $<\mathrm{b}>$ metafor $</ \mathrm{b}>$ Package. Fournal of Statistical Software, 36(3), 1-48. Retrieved from http://wwW . jstat soft. org/v36/i03/ doi: 10.18637/jss.v036 .i03

Williams, D. R., \& Bürkner, P. C. (2017). Effects of intranasal oxytocin on symptoms of schizophrenia: A multivariate Bayesian meta-analysis (Vol. 75). doi: 10.1016/j.psyneuen.2016.10 .013

Zhang, Y., \& Sutton, C. (2014). Semi-Separable Hamiltonian Monte Carlo for Inference in Bayesian Hierarchical Models. Neural Information Processing Systems, 10-18. 